\title{
ON THE KRALL-TYPE POLYNOMIALS
}

\author{
R. ÁLVAREZ-NODARSE, J. ARVESÚ, AND F. MARCELLÁN
}

Received 10 December 2003 and in revised form 6 July 2004

Using a general and simple algebraic approach, some results on Krall-type orthogonal polynomials and some of their extensions are obtained.

\section{Introduction}

Let $\mathfrak{u}: \mathbb{P} \mapsto \mathbb{R}$ be a quasidefinite linear functional in the vector space $\mathbb{P}$ of polynomials with complex coefficients, then there exists a sequence of monic polynomials $\left(P_{n}\right)_{n}$ with $\operatorname{deg} P_{n}=n$, such that [12]

$$
\left\langle\mathfrak{u}, P_{n} P_{m}\right\rangle=k_{n} \delta_{n, m}, \quad k_{n} \neq 0 .
$$

Special cases of quasidefinite linear functionals are the classical ones (those of Jacobi, Laguerre, Hermite, and Bessel).

In recent years perturbations of a quasidefinite linear functional $\mathfrak{u}$ via the addition of Dirac delta functionals and their derivatives have been extensively studied (see, e.g., $[1,5,14,15,16,17,18,19,23]$ and the references therein), that is, the linear functional

$$
\tilde{\mathfrak{u}}=\mathfrak{u}+\sum_{i=1}^{M} A_{i} \delta\left(x-a_{i}\right)-\sum_{j=1}^{N} B_{j} \delta^{\prime}\left(x-b_{j}\right),
$$

where $\left(A_{i}\right)_{i=1}^{M}$ and $\left(B_{j}\right)_{j=1}^{N}$ are real numbers, $\delta(x-y)$ and $\delta^{\prime}(x-y)$ mean the Dirac linear functional and its derivative, respectively, defined by $\langle\delta(x-y), p(x)\rangle=p(y),\left\langle\delta^{\prime}(x-\right.$ $y), p(x)\rangle=-p^{\prime}(y)$, for all $p \in \mathbb{P}$. If at most one $B_{i}$ is nonzero and $A_{i}=0, i=1, \ldots, m$, then the analysis of the quasi-definiteness of the linear functional $\tilde{u}$ is presented in [9].

Special important examples appear when $\mathfrak{u}$ is a classical linear functional and the mass points are located at the ends of the interval of orthogonality. We have studied the corresponding sequences of orthogonal polynomials in this case in several papers: for the Laguerre linear functional see [2, 3], for the Bessel linear functional see [7], for the Jacobi linear functional see [8], and for the Hermite case see [1]. In particular 
the quasi-definiteness of $\tilde{u}$, relative asymptotics, the representation as a hypergeometric function, and the location of their zeros have been obtained.

This kind of perturbations can be seen as the simplest (lower-order) discretecontinuous version of the "bispectral" property. The continuous "bispectral problem" consists of describing all Schrödinger-type differential operators of second order such that their eigenfunctions should satisfy a differential equation (of arbitrary finite order) in the spectral variable. The basic tools are based in the Darboux factorization method [13]. In the discrete-continuous case, this factorization is related to the LU and UL factorization of the Jacobi matrix associated with the sequence of orthogonal polynomials which are the eigenfunctions of a second-order linear differential operator of hypergeometric type. Thus the so-called Krall polynomials appear in a natural way [15, 16, 17, 18, 19].

Very recently, in [11], the study of sequences of orthogonal polynomials and their associated Hankel determinants when we add an extra term to a weight function is considered using the Heine formula. In particular, the authors analyze separately the addition of $A \delta(x-a)$ and $A \delta^{\prime}(x-a)$. Notice that they do not discuss the quasidefinite character of the linear functional $\tilde{\mathfrak{u}}$ given by either

$$
\tilde{\mathfrak{u}}=\mathfrak{u}+A \delta(x-a) \quad \text { or } \quad \tilde{\mathfrak{u}}=\mathfrak{u}-A \delta^{\prime}(x-a) .
$$

This is a very important gap, specially when derivatives of Dirac delta functionals are considered, because necessary and sufficient conditions for the existence of the corresponding sequence of orthogonal polynomials are needed. They obtain particular cases of our previous results $[2,3,4,5,8]$ using a different approach based in the above-mentioned Heine formula.

The aim of the present contribution is to cover the above gap about the quasidefiniteness when we add two extra terms to a linear functional $\mathfrak{u}$ in such a way that the perturbed linear functional is given by

$$
\tilde{\mathfrak{u}}=\mathfrak{u}+A \delta(x-a)-B \delta^{\prime}(x-b), \quad a \neq b .
$$

The case $a=b$ has been considered in some previous work by the authors and coworkers $[2,3,5,7]$.

We use an innovative and algebraic approach taking into account the orthogonality properties of the sequence of polynomials.

Section 2 deals with the analysis of necessary and sufficient conditions for the quasidefiniteness of $\tilde{\mathfrak{u}}$ given by (1.4). In such a case, the coefficients of the three-term recurrence relation that the corresponding sequence of monic orthogonal polynomials satisfies is obtained. Furthermore, if we assume that the $\mathfrak{u}$ is a semiclassical linear functional, that is, it satisfies a Pearson equation $D[\phi \mathfrak{u}]=\Psi \mathfrak{u}$, with $\phi, \psi$ polynomials such that $\operatorname{deg}(\psi) \geq 1$, see [24], then the linear functional $\tilde{\mathfrak{u}}$ is also semiclassical. This can be read in terms of the holonomic second-order linear differential equation that such polynomials satisfy. The explicit expression for their coefficients is given. Finally, as an application, the WKB method is used for the analysis of the location of their zeros. 


\section{General theory}

For the sake of simplicity we will consider a linear functional $\tilde{\mathfrak{u}}$ given by $\tilde{\mathfrak{u}}=\mathfrak{u}+A \delta(x-$ $a)-B \delta^{\prime}(x-b)$. Here we assume that $a$ is different from $b$. This situation has been extensively considered in the literature for $a=b$ and was found to yield a simplification of the computation in the representation formulas (see, e.g., [1]). Thus we will recover the result in [11] in a very simple way (for the general case we refer the reader to [1]).

2.1. Representation formulas. If $\tilde{\mathfrak{u}}$ is quasidefinite, then there exists a sequence of monic polynomials $\left(\widetilde{P}_{n}\right)_{n}$ orthogonal with respect to $\tilde{\mathfrak{u}}$, and therefore, we can consider the Fourier expansion

$$
\widetilde{P}_{n}(x)=P_{n}(x)+\sum_{k=0}^{n-1} \lambda_{n, k} P_{k}(x)
$$

Thus, for $0 \leq k \leq n-1$,

$$
\begin{aligned}
\lambda_{n, k} & =\frac{\left\langle\mathfrak{u}, \widetilde{P}_{n}(x) P_{k}(x)\right\rangle}{\left\langle\mathfrak{u}, P_{k}^{2}(x)\right\rangle}=-\frac{A \widetilde{P}_{n}(a) P_{k}(a)+B \widetilde{P}_{n}(b) P_{k}^{\prime}(b)+B \widetilde{P}_{n}^{\prime}(b) P_{k}(b)}{\left\langle\mathfrak{u}, P_{k}^{2}(x)\right\rangle} \\
& =A \widetilde{P}_{n}(a) \frac{P_{k}(a)}{\left\langle\mathfrak{u}, P_{k}^{2}(x)\right\rangle}-B \widetilde{P}_{n}(b) \frac{P_{k}^{\prime}(b)}{\left\langle\mathfrak{u}, P_{k}^{2}(x)\right\rangle}-B \widetilde{P}_{n}^{\prime}(b) \frac{P_{k}(b)}{\left\langle\mathfrak{u}, P_{k}^{2}(x)\right\rangle} .
\end{aligned}
$$

As a consequence, (2.1) becomes

$$
\widetilde{P}_{n}(x)=P_{n}(x)-A \widetilde{P}_{n}(a) \mathrm{K}_{n-1}(x, a)-B \widetilde{P}_{n}(b) \mathrm{K}_{n-1}^{(0,1)}(x, b)-B \widetilde{P}_{n}^{\prime}(b) \mathrm{K}_{n-1}(x, b),
$$

where, as usual,

$$
\mathrm{K}_{n}^{(i, j)}(x, y)=\sum_{l=0}^{n} \frac{P_{l}^{(i)}(x) P_{l}^{(j)}(y)}{\left\langle\mathfrak{u}, P_{l}^{2}(x)\right\rangle}, \quad P_{l}^{(i)}(x):=\frac{d^{i}}{d x^{i}} P_{l}(x), \quad i, j \in \mathbb{N} .
$$

As a convention, $\mathrm{K}_{n}^{(0,0)}(x, y):=\mathrm{K}_{n}(x, y)$ denotes the reproducing kernel associated with the linear functional $\mathfrak{u}$. It is very well known (reproducing property of the kernels) that $\left\langle\mathfrak{u}, \mathrm{K}_{n}(x, y) p(x)\right\rangle=p(y)$ for every polynomial $p(x)$ of degree less than or equal to $n$. Evaluating (2.3) for $x=a$, we get

$$
\widetilde{P}_{n}(a)=P_{n}(a)-A \widetilde{P}_{n}(a) \mathrm{K}_{n-1}(a, a)-B \widetilde{P}_{n}(b) \mathrm{K}_{n-1}^{(0,1)}(a, b)-B \widetilde{P}_{n}^{\prime}(b) \mathrm{K}_{n-1}(a, b) .
$$

A similar evaluation in (2.3) for $x=b$ gives

$$
\widetilde{P}_{n}(b)=P_{n}(b)-A \widetilde{P}_{n}(a) \mathrm{K}_{n-1}(b, a)-B \widetilde{P}_{n}(b) \mathrm{K}_{n-1}^{(0,1)}(b, b)-B \tilde{P}_{n}^{\prime}(b) \mathrm{K}_{n-1}(b, b) .
$$

Finally, taking derivatives in (2.3) and evaluating the resulting expression for $x=b$, we obtain

$$
\widetilde{P}_{n}^{\prime}(b)=P_{n}^{\prime}(b)-A \widetilde{P}_{n}(a) \mathrm{K}_{n-1}^{(1,0)}(b, a)-B \widetilde{P}_{n}(b) \mathrm{K}_{n-1}^{(1,1)}(b, b)-B \widetilde{P}_{n}^{\prime}(b) \mathrm{K}_{n-1}^{(1,0)}(b, b) .
$$


Thus we get a system of three linear equations in the variables $\widetilde{P}_{n}(a), \widetilde{P}_{n}(b)$, and $\widetilde{P}_{n}^{\prime}(b)$ :

$$
\left(\begin{array}{c}
\widetilde{P}_{n}(a) \\
\widetilde{P}_{n}(b) \\
\widetilde{P}_{n}^{\prime}(b)
\end{array}\right)=\left(\begin{array}{c}
P_{n}(a) \\
P_{n}(b) \\
P_{n}^{\prime}(b)
\end{array}\right)-\mathbb{K}_{n-1} \mathrm{D}\left(\begin{array}{c}
\widetilde{P}_{n}(a) \\
\widetilde{P}_{n}(b) \\
\widetilde{P}_{n}^{\prime}(b)
\end{array}\right)
$$

where

$$
\mathrm{D}=\left(\begin{array}{ccc}
A & 0 & 0 \\
0 & 0 & B \\
0 & B & 0
\end{array}\right), \quad \mathbb{K}_{n-1}=\left(\begin{array}{ccc}
\mathrm{K}_{n-1}(a, a) & \mathrm{K}_{n-1}(a, b) & \mathrm{K}_{n-1}^{(0,1)}(a, b) \\
\mathrm{K}_{n-1}(b, a) & \mathrm{K}_{n-1}(b, b) & \mathrm{K}_{n-1}^{(0,1)}(b, b) \\
\mathrm{K}_{n-1}^{(1,0)}(b, a) & \mathrm{K}_{n-1}^{(0,1)}(b, b) & \mathrm{K}_{n-1}^{(1,1)}(b, b)
\end{array}\right) .
$$

Notice that if the matrix $\mathrm{I}+\mathbb{K}_{n-1} \mathrm{D}$, where $\mathrm{I}$ is the identity matrix, is nonsingular, then we get the existence and uniqueness for the solution of (2.8). Therefore, (2.3) becomes

$$
\begin{aligned}
\widetilde{P}_{n}(x)= & P_{n}(x)-\left(\mathrm{K}_{n-1}(x, a), \mathrm{K}_{n-1}(x, b), \mathrm{K}_{n-1}^{(0,1)}(x, a)\right) \\
& \times \mathrm{D}\left(\mathrm{I}+\mathbb{K}_{n-1} \mathrm{D}\right)^{-1}\left(P_{n}(a), P_{n}(b), P_{n}^{\prime}(b)\right)^{T} .
\end{aligned}
$$

All the above lead to the following theorem.

THEOREm 2.1. The linear functional $\tilde{\mathfrak{u}}$ is a quasidefinite linear functional if and only if

(i) the matrix $\mathrm{I}+\mathbb{K}_{n-1} \mathrm{D}$ is nonsingular for every $n \in \mathbb{N}$,

(ii) $\left\langle\mathfrak{u}, P_{n}^{2}(x)\right\rangle+\left(P_{n}(a), P_{n}(b), P_{n}^{\prime}(b)\right) \mathrm{D}\left(\mathrm{I}+\mathbb{K}_{n-1} \mathrm{D}\right)^{-1}\left(\begin{array}{l}P_{n}(a) \\ P_{n}(b) \\ P_{n}^{\prime}(b)\end{array}\right) \neq 0$ for every $n \in \mathbb{N}$,

(iii) the norm $\left\langle\tilde{\mathfrak{u}}, \widetilde{P}_{n}^{2}(x)\right\rangle$ is given by

$$
\begin{aligned}
\left\langle\tilde{\mathfrak{u}}, \widetilde{P}_{n}^{2}(x)\right\rangle= & \left\langle\mathfrak{u}, P_{n}^{2}(x)\right\rangle+\left(P_{n}(a), P_{n}(b), P_{n}^{\prime}(b)\right) \\
& \times \mathrm{D}\left(\mathrm{I}+\mathbb{K}_{n-1} \mathrm{D}\right)^{-1}\left(P_{n}(a), P_{n}(b), P_{n}^{\prime}(b)\right)^{T} \neq 0 .
\end{aligned}
$$

In such a case, the sequence $\left(\widetilde{P}_{n}\right)_{n}$ of monic orthogonal polynomials with respect to $\tilde{\mathfrak{u}}$ is given by (2.10).

Formula (2.11) constitutes the first representation formula for the polynomials $\left(\widetilde{P}_{n}\right)_{n}$. Remark 2.2. If the entries of the matrix $\mathrm{D}$ are nonzero, then $\mathrm{D}$ is a nonsingular Hermitian matrix, and

$$
\mathrm{D}\left(\mathrm{I}+\mathbb{K}_{n-1} \mathrm{D}\right)^{-1}=\left(\mathrm{D}^{-1}+\mathbb{K}_{n-1}\right)^{-1}=\mathrm{M}_{n-1},
$$

where $\mathrm{D}^{-1}+\mathbb{K}_{n-1}$ is a Hermitian matrix. Therefore (2.11) means that

$$
\begin{aligned}
& 1+\varepsilon_{n}\left(\hat{P}_{n}(a), \hat{P}_{n}(b), \hat{P}_{n}^{\prime}(b)\right) \mathrm{M}_{n-1}\left(\begin{array}{c}
\hat{P}_{n}(a) \\
\hat{P}_{n}(b) \\
\hat{P}_{n}^{\prime}(b)
\end{array}\right) \neq 0, \quad \hat{P}_{n}\left(a_{i}\right)=\frac{P_{n}\left(a_{i}\right)}{\sqrt{\left|\left\langle\mathfrak{u}, P_{n}^{2}(x)\right\rangle\right|}}, \\
& a_{i}=a, b \text {, and } \varepsilon_{n}=\operatorname{sign}\left(\left\langle\mathfrak{u}, P_{n}^{2}(x)\right\rangle\right) .
\end{aligned}
$$


We now obtain another representation formula. Taking into account the ChristoffelDarboux formula

$$
\mathrm{K}_{n-1}(x, y)=\frac{1}{k_{n}}\left[\frac{P_{n}(x) P_{n-1}(y)-P_{n}(y) P_{n-1}(x)}{x-y}\right], \quad k_{n}=\left\langle\mathfrak{u}, P_{n}^{2}(x)\right\rangle,
$$

as well as

$$
\mathrm{K}_{n-1}^{(0,1)}(x, y)=\frac{1}{k_{n}}\left[\frac{P_{n}(x) P_{n-1}^{\prime}(y)-P_{n}^{\prime}(y) P_{n-1}(x)}{x-y}+\frac{P_{n}(x) P_{n-1}(y)-P_{n}(y) P_{n-1}(x)}{(x-y)^{2}}\right],
$$

inserting these two expressions in (2.10), and denoting $\phi(x)=(x-a)(x-b)^{2}$, we deduce

$$
\phi(x) \widetilde{P}_{n}(x)=A(x ; n) P_{n}(x)+B(x ; n) P_{n-1}(x),
$$

where $A(x ; n)$ and $B(x ; n)$ are polynomials of degrees independent of $n$, of at most 3 and 2 , respectively. On the other hand, from the three-term recurrence relation that the sequence $\left(P_{n}\right)_{n}$ satisfies

$$
x P_{n}(x)=P_{n+1}(x)+\beta_{n} P_{n}(x)+\gamma_{n} P_{n-1}(x), \quad \gamma_{n} \neq 0, \forall n \in \mathbb{N},
$$

and taking into account (2.16), for $n \geq 1$, we get

$$
\begin{gathered}
\phi(x) \tilde{P}_{n}(x)=C(x ; n) P_{n+1}(x)+D(x ; n) P_{n}(x), \\
C(x ; n)=-\frac{B(x ; n)}{\gamma_{n}}, \quad D(x ; n)=A(x ; n)+\frac{x-\beta_{n}}{\gamma_{n}} B(x ; n) .
\end{gathered}
$$

Now, from any of the above expressions a very useful representation formula follows. In fact, if $\mathfrak{u}$ is a semiclassical linear functional [24], then there exist a polynomial $\psi(x)$ and two polynomials $M_{1}(x ; n), N_{1}(x ; n)$ with degree independent of $n$ such that

$$
\psi(x) P_{n}^{\prime}(x)=M_{1}(x ; n) P_{n}(x)+N_{1}(x ; n) P_{n-1}(x) ;
$$

or, equivalently, using the three-term recurrence relation (TTRR) (2.17), we get

$$
\psi(x) P_{n}^{\prime}(x)=M_{2}(x ; n) P_{n}(x)+N_{2}(x ; n) P_{n+1}(x),
$$

where again the degrees of $M_{2}(x ; n)$ and $N_{2}(x ; n)$ are independent of $n$. Usually formulas (2.19) and (2.20) represent the lowering and raising operators for the semiclassical family $\left(P_{n}\right)_{n}$.

Using (2.16) and (2.19) or (2.18) and (2.20), we obtain the formula

$$
q(x ; n) \widetilde{P}_{n}(x)=a(x ; n) P_{n}(x)+b(x ; n) P_{n}^{\prime}(x),
$$

where $a, b$, and $q$ are polynomials with degree independent of $n$. The last formula plays a fundamental role in finding the second-order linear differential equation that the polynomials $\left(\widetilde{P}_{n}\right)_{n}$ satisfy, as shown in Section 2.3 . 
2.2. Three-term recurrence relation and lowering and raising operators. In the following we assume that $\tilde{\mathfrak{u}}$ is quasidefinite. Then, the sequence $\left(\widetilde{P}_{n}\right)_{n}$ of monic polynomials orthogonal with respect to $\tilde{\mathfrak{u}}$ satisfies a TTRR:

$$
x \widetilde{P}_{n}(x)=\widetilde{P}_{n+1}(x)+\widetilde{\beta}_{n} \widetilde{P}_{n}(x)+\widetilde{\gamma}_{n} \widetilde{P}_{n-1}(x), \quad n \in \mathbb{N},
$$

with the initial conditions $\widetilde{P}_{-1}(x)=0, \widetilde{P}_{0}(x)=1$.

Here we will obtain the coefficients $\widetilde{\beta}_{n}$ and $\tilde{\gamma}_{n}$ of the TTRR (2.22) for the polynomials $\widetilde{P}_{n}$ orthogonal with respect to $\tilde{\mathfrak{u}}$, in terms of the coefficients $\beta_{n}$ and $\gamma_{n}$ of the TTRR (2.17) of the monic polynomials orthogonal with respect to $\mathfrak{u}$. Since

$$
\tilde{\gamma}_{n}=\frac{\left\langle\tilde{\mathfrak{u}}, \widetilde{P}_{n}^{2}(x)\right\rangle}{\left\langle\tilde{\mathfrak{u}}, \widetilde{P}_{n-1}^{2}(x)\right\rangle},
$$

then, taking into account (2.11) as well as Remark 2.2 of Theorem 2.1, for $n>1$, we get

$$
\tilde{\gamma}_{n}=\gamma_{n} \frac{1+\varepsilon_{n}\left(\hat{P}_{n}(a), \hat{P}_{n}(b), \hat{P}_{n}^{\prime}(b)\right) \mathrm{M}_{n-1}\left(\hat{P}_{n}(a), \hat{P}_{n}(b), \hat{P}_{n}^{\prime}(b)\right)^{T}}{1+\varepsilon_{n-1}\left(\hat{P}_{n-1}(a), \hat{P}_{n-1}(b), \hat{P}_{n-1}^{\prime}(b)\right) \mathrm{M}_{n-2}\left(\hat{P}_{n-1}(a), \hat{P}_{n-1}(b), \hat{P}_{n-1}^{\prime}(b)\right)^{T}},
$$

as well as, for $n=1$,

$$
\begin{aligned}
\tilde{\gamma}_{1} & =\frac{\left\langle\widetilde{\mathfrak{u}}, \widetilde{P}_{1}^{2}(x)\right\rangle}{\left\langle\tilde{\mathfrak{u}}, \widetilde{P}_{0}^{2}(x)\right\rangle}=\frac{\left\langle\tilde{\mathfrak{u}}, \widetilde{P}_{1}^{2}(x)\right\rangle}{\left\langle\mathfrak{u}, \widetilde{P}_{0}^{2}(x)\right\rangle+\sum_{i=1}^{M} A_{i}} \\
& =\gamma_{1} \frac{1+\varepsilon_{1}\left(\hat{P}_{1}(a), \hat{P}_{1}(b), \hat{P}_{1}^{\prime}(b)\right) \mathrm{M}_{0}\left(\hat{P}_{1}(a), \hat{P}_{1}(b), \hat{P}_{1}^{\prime}(b)\right)^{T}}{1+A / u_{0}}
\end{aligned}
$$

where $u_{0}=\langle\mathfrak{u}, 1\rangle$ is the first moment of the linear functional $\mathfrak{u}$.

On the other hand, $\widetilde{\beta}_{n}=\left\langle\widetilde{\mathfrak{u}}, x \widetilde{P}_{n}^{2}(x)\right\rangle /\left\langle\widetilde{\mathfrak{u}}, \widetilde{P}_{n}^{2}(x)\right\rangle$. Nevertheless, it is more convenient to compute $\tilde{\beta}_{n}$ in a different way. If $\tilde{b}_{n}$ denote the coefficient of $x^{n-1}$ for $\widetilde{P}_{n}$ and $b_{n}$ the corresponding coefficient of $x^{n-1}$ for $P_{n}$, then we have, $\tilde{\beta}_{n}=\tilde{b}_{n}-\tilde{b}_{n+1}$. To obtain $\tilde{b}_{n}$ we use (2.10):

$$
\tilde{b}_{n}=b_{n}-\varepsilon_{n} \varepsilon_{n-1}\left|\gamma_{n}\right|^{1 / 2}\left(\widehat{P}_{n-1}(a), \hat{P}_{n-1}(b), \hat{P}_{n-1}^{\prime}(b)\right) \mathrm{M}_{n-1}\left(\hat{P}_{n}(a), \hat{P}_{n}(b), \hat{P}_{n}^{\prime}(b)\right)^{T}
$$

Thus, for $n \geq 1$,

$$
\begin{aligned}
\tilde{\beta}_{n}= & \beta_{n}+\varepsilon_{n} \varepsilon_{n+1}\left|\gamma_{n+1}\right|^{1 / 2}\left(\hat{P}_{n}(a), \hat{P}_{n}(b), \hat{P}_{n}^{\prime}(b)\right) \mathrm{M}_{n}\left(\hat{P}_{n+1}(a), \hat{P}_{n+1}(b), \hat{P}_{n+1}^{\prime}(b)\right)^{T} \\
& -\varepsilon_{n} \varepsilon_{n-1}\left|\gamma_{n}\right|^{1 / 2}\left(\hat{P}_{n-1}(a), \hat{P}_{n-1}(b), \hat{P}_{n-1}^{\prime}(b)\right) \mathrm{M}_{n-1}\left(\hat{P}_{n}(a), \hat{P}_{n}(b), \hat{P}_{n}^{\prime}(b)\right)^{T} .
\end{aligned}
$$


Finally, for $n=0$, we have

$$
\tilde{\beta}_{0}=\frac{\langle\tilde{\mathfrak{u}}, x\rangle}{\langle\tilde{\mathfrak{u}}, 1\rangle}=\frac{u_{1}+a A+B}{u_{0}+A}
$$

Notice that in order to compute the values $\tilde{b}_{n}$ we can also use (2.21).

Following the ideas in [1] it is easy to show that in this case the polynomials $\widetilde{P}_{n}$ admit both lowering and raising operators of the form

$$
\begin{aligned}
& \phi^{2}(x) \psi(x) \widetilde{P}_{n}^{\prime}(x)=\widetilde{M}_{1}(x ; n) \widetilde{P}_{n}(x)+\tilde{N}_{1}(x ; n) \widetilde{P}_{n-1}(x), \\
& \phi^{2}(x) \psi(x) \widetilde{P}_{n}^{\prime}(x)=\widetilde{M}_{2}(x ; n) \widetilde{P}_{n}(x)+\tilde{N}_{2}(x ; n) \widetilde{P}_{n+1}(x),
\end{aligned}
$$

where $\widetilde{M}_{1}(x ; n), \widetilde{N}_{1}(x ; n), \widetilde{M}_{2}(x ; n)$, and $\widetilde{M}_{2}(x ; n)$ are polynomials depending on $n$ and of bounded degree independent of $n$, respectively. These two formulas are very useful. In particular, from them we can easily derive the second-order linear differential equation that the polynomials $\widetilde{P}_{n}$ satisfy (see [1] for more details). Nevertheless, from formula (2.21), it is possible to find it by a simpler method as we will show in the next section. In particular this leads to the second-order linear differential equation that the polynomials considered in $[2,3,7,8]$, and therefore [11], satisfy.

2.3. Second-order linear differential equation for $\left(\widetilde{P}_{n}\right)_{n}$. Here we will describe an algorithm for finding the second-order differential equations (SODE) which satisfy the polynomials $\widetilde{P}_{n}(x)$ defined by $(2.21)$, where $\left(P_{n}\right)_{n}$ is a solution of the SODE

$$
\sigma(x) P_{n}^{\prime \prime}(x)+\tau(x) P_{n}^{\prime}(x)+\lambda(x) P_{n}(x)=0 .
$$

A special case is when $\left(P_{n}\right)_{n}$ constitutes a classical or semiclassical orthogonal family so (2.30) takes place. Taking derivatives in (2.21), we get

$$
\begin{aligned}
& q^{\prime}(x ; n) \widetilde{P}_{n}(x)+q(x ; n) \tilde{P}_{n}^{\prime}(x) \\
& \quad=a^{\prime}(x ; n) P_{n}(x)+a(x ; n) P_{n}^{\prime}(x)+b^{\prime}(x ; n) P_{n}^{\prime}(x)+b(x ; n) P_{n}^{\prime \prime}(x) .
\end{aligned}
$$

Now, multiplying the above formula by $\sigma(x)$ and using the SODE for the polynomials $\left(P_{n}\right)_{n}$, we can rewrite $b(x ; n) P_{n}^{\prime \prime}(x)$ as a combination of $P_{n}(x)$ and $P_{n}^{\prime}(x)$, with polynomial coeffcients. To eliminate the term $q^{\prime}(x ; n) \widetilde{P}_{n}(x)$, we can multiply by $q$ and use $(2.21)$. These two operations yield

$$
r(x ; n) \widetilde{P}_{n}^{\prime}(x)=c(x ; n) P_{n}(x)+d(x ; n) P_{n}^{\prime}(x),
$$

where

$$
\begin{aligned}
& r(x ; n)=\sigma(x) q^{2}(x ; n), \\
& c(x ; n)=q(x ; n) \sigma(x) a^{\prime}(x)-b(x ; n) q(x ; n) \lambda(x)-\sigma(x) a(x ; n) q^{\prime}(x ; n), \\
& d(x ; n)=q(x ; n) \sigma(x) a(x ; n)+q(x ; n) \sigma(x) b^{\prime}(x)-b(x ; n) q(x ; n) \tau(x)-\sigma(x) b(x ; n) q^{\prime}(x ; n) .
\end{aligned}
$$


Analogously, taking derivatives in (2.32) and using the SODE (2.30), we obtain

$$
s(x ; n) \tilde{P}_{n}^{\prime \prime}(x)=e(x ; n) P_{n}(x)+f(x ; n) P_{n}^{\prime}(x),
$$

with

$$
\begin{aligned}
& s(x ; n)=\sigma(x) r^{2}(x ; n), \\
& e(x ; n)=r(x ; n) \sigma(x) c^{\prime}(x)-d(x ; n) r(x ; n) \lambda(x)-\sigma(x) c(x ; n) r^{\prime}(x ; n), \\
& f(x ; n)=r(x ; n) \sigma(x) c(x ; n)+r(x ; n) \sigma(x) d^{\prime}(x)-d(x ; n) r(x) \tau(x)-\sigma(x) d(x ; n) r^{\prime}(x ; n) .
\end{aligned}
$$

In formulas (2.32), (2.34), $r, s, c, d, e$, and $f$ are polynomials of bounded degree with coefficients depending, in general, on $n$ (they depend only on the functions $\sigma(x), \tau(x)$, $\lambda(x), q(x ; n), a(x ; n)$, and $b(x ; n)$ which are polynomials on $x)$. The expressions (2.21), (2.32), and (2.34) yield

$$
\left|\begin{array}{lll}
q(x ; n) \widetilde{P}_{n}(x) & a(x ; n) & b(x ; n) \\
r(x ; n) \widetilde{P}_{n}^{\prime}(x) & c(x ; n) & d(x ; n) \\
s(x ; n) \widetilde{P}_{n}^{\prime \prime}(x) & e(x ; n) & f(x ; n)
\end{array}\right|=0
$$

where the functions $q(x ; n), a(x ; n)$, and $b(x ; n)$ are known from expression $(2.21)$, and $c(x ; n), d(x ; n), e(x ; n), f(x ; n), r(x)$, and $s(x ; n)$ can be found from (2.33) and (2.35). Expanding the determinant in (2.36) by the first column, we get

$$
\tilde{\sigma}_{n}(x) \tilde{P}_{n}^{\prime \prime}(x)+\tilde{\tau}_{n}(x) \widetilde{P}_{n}^{\prime}(x)+\tilde{\lambda}_{n}(x) \tilde{P}_{n}(x)=0,
$$

where

$$
\begin{aligned}
& \tilde{\sigma}_{n}(x)=s(x ; n)[a(x ; n) d(x ; n)-c(x ; n) b(x ; n)], \\
& \tilde{\tau}_{n}(x)=r(x ; n)[e(x ; n) b(x ; n)-a(x ; n) f(x ; n)], \\
& \tilde{\lambda}_{n}(x)=q(x ; n)[c(x ; n) f(x ; n)-e(x ; n) d(x ; n)] .
\end{aligned}
$$

A straightforward calculation shows us that all coefficients in (2.37) have a common factor $q^{4}(x ; n) \sigma^{2}(x)$. Then $(2.38)$ can be rewritten as

$$
\begin{aligned}
& \tilde{\sigma}_{n}(x)=s(x ; n)\left[\frac{a(x ; n) d(x ; n)-c(x ; n) b(x ; n)}{q^{4}(x ; n) \sigma^{2}(x)}\right], \\
& \tilde{\tau}_{n}(x)=r(x ; n)\left[\frac{e(x ; n) b(x ; n)-a(x ; n) f(x ; n)}{q^{4}(x ; n) \sigma^{2}(x)}\right], \\
& \tilde{\lambda}_{n}(x)=q(x ; n)\left[\frac{c(x ; n) f(x ; n)-e(x ; n) d(x ; n)}{q^{4}(x) \sigma^{2}(x)}\right] .
\end{aligned}
$$


To conclude this section, we point out that special cases of the above algorithm have been extensively used for getting the differential equations of the Krall-type polynomials, Sobolev-type polynomials, and so forth (see, e.g., $[2,4,5,7,8,20,21,22]$ and the references therein).

2.4. Density of zeros. From the SODE (2.37) the density of the distribution of zeros immediately follows. In fact, using the WKB approximation (for more details about this approach, we refer to the papers $[6,25]$ and the references therein), we have the following theorem.

THEOREM 2.3. Let $S$ and $\epsilon$ be such that

$$
\begin{aligned}
& S(x)=\frac{1}{4 \tilde{\sigma}_{n}^{2}(x)}\left\{2 \tilde{\sigma}_{n}(x)\left[2 \tilde{\lambda}_{n}(x)-\tilde{\tau}_{n}^{\prime}(x)\right]+\tilde{\tau}_{n}(x)\left[2 \tilde{\sigma}_{n}^{\prime}(x)-\tilde{\tau}_{n}(x)\right]\right\}, \\
& \epsilon(x)=\frac{1}{4[S(x)]^{2}}\left\{\frac{5\left[S^{\prime}(x)\right]^{2}}{4[S(x)]}-S^{\prime \prime}(x)\right\}=\frac{P(x, n)}{Q(x, n)},
\end{aligned}
$$

where $P(x, n)$ and $Q(x, n)$ are polynomials in $x$ as well as in $n$. If the condition $\epsilon(x) \ll 1$ holds, then the semiclassical or WKB density of zeros of the solutions of (2.37) is

$$
\rho_{\mathrm{WKB}}(x)=\frac{1}{\pi} \sqrt{S(x)}, \quad x \in I \subseteq \mathbb{R}
$$

in every interval I where the function $S$ is positive.

The above theorem has been extensively used in the study of the asymptotic distribution of zeros of the Krall-type polynomials [5] as well as their extensions [3, 8]. We mention also that in all cases under consideration, for $n$ large enough, we get $\epsilon(x) \sim n^{-1}$. Then, from the above theorem, the corresponding WKB density of zeros of the polynomials $\left(\widetilde{P}_{n}\right)_{n}$ follows. The computations are very hard and cumbersome so the symbolic package Mathematica is again a very useful tool (see $[3,5,8]$ ). Finally, we point out that from the SODE the moments of the zero distribution easily follows using the approach described in [10].

\section{Acknowledgments}

This work was partially supported by Dirección General de Investigación (Ministerio de Ciencia y Tecnología) of Spain BFM 2003-06335-C03, Junta de Andalucía FQM-0262 (RAN), and INTAS Research Network NeCCA (INTAS 03-51-6637) (FM and JA). We also thank the referees for their comments and suggestions in order to improve our manuscript.

\section{References}

[1] R. Álvarez-Nodarse, J. Arvesú, and F. Marcellán, Modifications of quasi-definite linear functionals via addition of delta and derivatives of delta Dirac functions, Indag. Math. (N.S.) 15 (2004), no. 1, 1-20.

[2] R. Álvarez-Nodarse and F. Marcellán, A generalization of the classical Laguerre polynomials, Rend. Circ. Mat. Palermo (2) 44 (1995), no. 2, 315-329. 

, A generalization of the class Laguerre polynomials: asymptotic properties and zeros, Appl. Anal. 62 (1996), no. 3-4, 349-366.

[4] - Limit relations between generalized orthogonal polynomials, Indag. Math. (N.S.) 8 (1997), no. 3, 295-316.

[5] R. Álvarez-Nodarse, F. Marcellán, and J. Petronilho, WKB approximation and Krall-type orthogonal polynomials, Acta Appl. Math. 54 (1998), no. 1, 27-58.

[6] E. R. Arriola, A. Zarzo, and J. S. Dehesa, Spectral properties of the biconfluent Heun differential equation, J. Comput. Appl. Math. 37 (1991), no. 1-3, 161-169.

[7] J. Arvesú, R. Álvarez-Nodarse, F. Marcellán, and K. H. Kwon, Some extension of the Bessel-type orthogonal polynomials, Integral Transform. Spec. Funct. 7 (1998), no. 3-4, 191-214.

[8] J. Arvesú, F. Marcellán, and R. Alvarez-Nodarse, On a modification of the Jacobi linear functional: asymptotic properties and zeros of the corresponding orthogonal polynomials, Acta Appl. Math. 71 (2002), no. 2, 127-158.

[9] S. Belmehdi and F. Marcellán, Orthogonal polynomials associated with some modifications of a linear functional, Appl. Anal. 46 (1992), no. 1-2, 1-24.

[10] E. Buendía, J. S. Dehesa, and F. J. Gálvez, The distribution of zeros of the polynomial eigenfunctions of ordinary differential operators of arbitrary order, Orthogonal Polynomials and Their Applications (Segovia, 1986) (M. Alfaro, J. S. Dehesa, F. J. Marcellán, J. L. Rubio de Francia, and J. Vinuesa, eds.), Lecture Notes in Math., vol. 1329, Springer, Berlin, 1988, pp. 222235.

[11] Y. Chen and J. Griffin, Krall-type polynomials via the Heine formula, J. Phys. A 35 (2002), no. 3, 637-656.

[12] T. S. Chihara, An Introduction to Orthogonal Polynomials, Gordon and Breach Science Publishers, New York, 1978.

[13] J. J. Duistermaat and F. A. Grünbaum, Differential equations in the spectral parameter, Comm. Math. Phys. 103 (1986), no. 2, 177-240.

[14] W. N. Everitt, K. H. Kwon, L. L. Littlejohn, and R. Wellman, Orthogonal polynomial solutions of linear ordinary differential equations, J. Comput. Appl. Math. 133 (2001), no. 1-2, 85-109.

[15] F. A. Grünbaum and L. Haine, Orthogonal polynomials satisfying differential equations: the role of the Darboux transformation, Symmetries and Integrability of Difference Equations (Estérel, PQ, 1994), CRM Proc. Lecture Notes, vol. 9, American Mathematical Society, Rhode Island, 1996, pp. 143-154.

[16] Bispectral Darboux transformations: an extension of the Krall polynomials, Int. Math. Res. Not. (1997), no. 8, 359-392.

[17] F. A. Grünbaum, L. Haine, and E. Horozov, On the Krall-Hermite and the Krall-Bessel polynomials, Int. Math. Res. Not. (1997), no. 19, 953-966.

[18] Some functions that generalize the Krall-Laguerre polynomials, J. Comput. Appl. Math. 106 (1999), no. 2, 271-297.

[19] L. Haine, The Bochner-Krall problem: some new perspectives, Special Functions 2000: Current Perspective and Future Directions (Tempe, Ariz) (J. Bustoz, M. E. H. Ismail, and S. K. Suslov, eds.), NATO Sci. Ser. II Math. Phys. Chem., vol. 30, Kluwer Academic Publishers, Dordrecht, 2001, pp. 141-178.

[20] J. Koekoek and R. Koekoek, On a differential equation for Koornwinder's generalized Laguerre polynomials, Proc. Amer. Math. Soc. 112 (1991), no. 4, 1045-1054.

[21] R. Koekoek, Differential equations for symmetric generalized ultraspherical polynomials, Trans. Amer. Math. Soc. 345 (1994), no. 1, 47-72.

[22] R. Koekoek and H. G. Meijer, A generalization of Laguerre polynomials, SIAM J. Math. Anal. 24 (1993), no. 3, 768-782. 
[23] K. H. Kwon, G. J. Yoon, and L. L. Littlejohn, Bochner-Krall orthogonal polynomials, Special Functions (Hong Kong, 1999) (C. Dunkl, M. Ismail, and R. Wong, eds.), World Scientific Publishing, New Jersey, 2000, pp. 181-193.

[24] P. Maroni, Une théorie algébrique des polynômes orthogonaux. Application aux polynômes orthogonaux semi-classiques [An algebraic theory of orthogonal polynomials. Application to semiclassical orthogonal polynomials], Orthogonal Polynomials and Their Applications (Erice, 1990) (C. Brezinski, L. Gori, and A. Ronveaux, eds.), IMACS Ann. Comput. Appl. Math., vol. 9, Baltzer, Basel, 1991, pp. 95-130.

[25] A. Zarzo and J. S. Dehesa, Spectral properties of solutions of hypergeometric-type differential equations, J. Comput. Appl. Math. 50 (1994), no. 1-3, 613-623.

R. Álvarez-Nodarse: Departamento de Análisis Matemático, Universidad de Sevilla, Apartado de Correos 1160, 41080 Sevilla, Spain; Instituto Carlos I de Física Teórica y Computacional, Universidad de Granada, 18071 Granada, Spain

E-mail address: ran@us.es

J. Arvesú: Departamento de Matemáticas, Universidad Carlos III de Madrid, Avenida de la Universidad 30, 28911 Leganés, Madrid, Spain

E-mail address: jarvesu@math.uc3m.es

F. Marcellán: Departamento de Matemáticas, Universidad Carlos III de Madrid, Avenida de la Universidad 30, 28911 Leganés, Madrid, Spain

E-mail address: pacomarc@ing.uc3m.es 


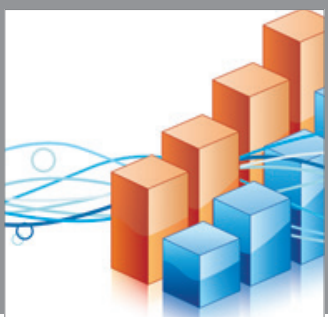

Advances in

Operations Research

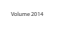

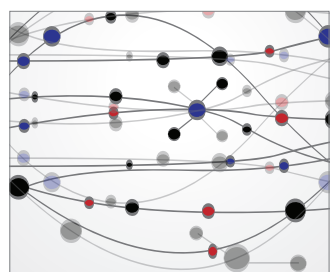

\section{The Scientific} World Journal
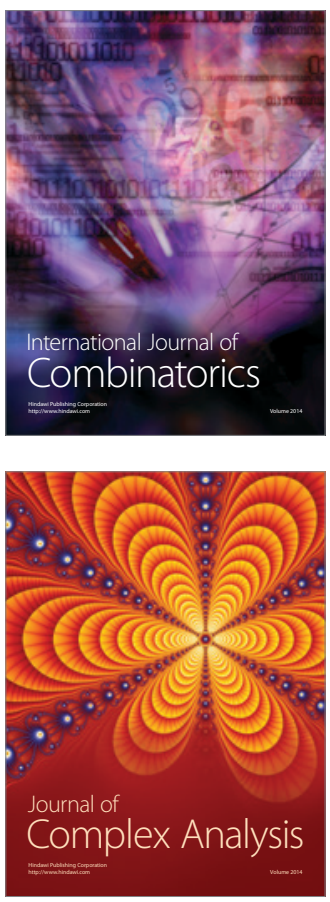

International Journal of

Mathematics and

Mathematical

Sciences
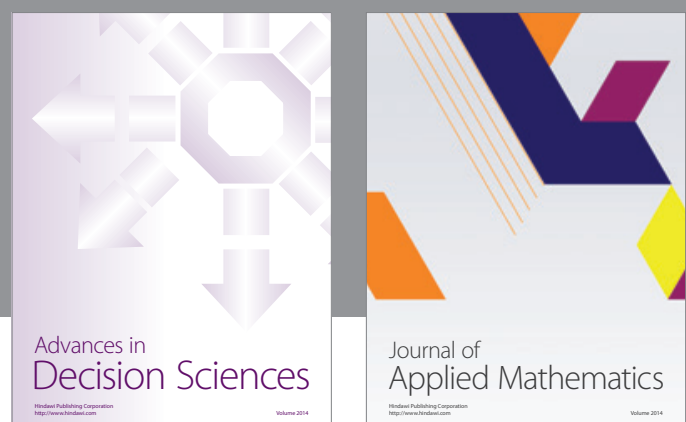

Journal of

Applied Mathematics
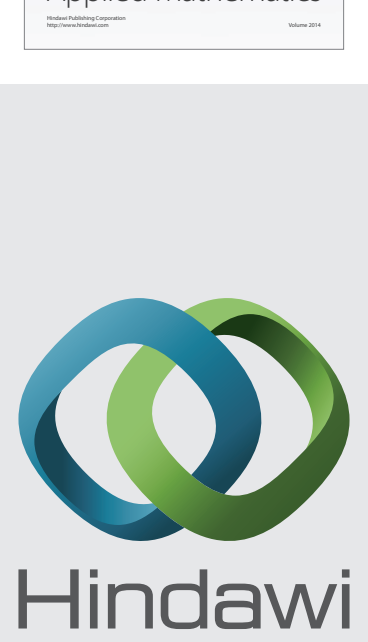

Submit your manuscripts at http://www.hindawi.com
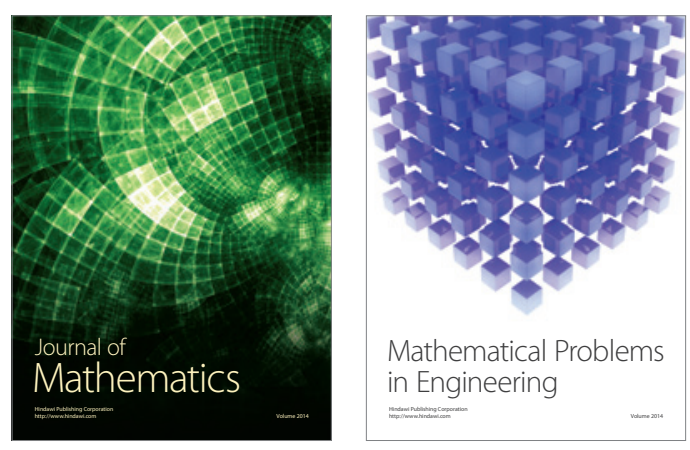

Mathematical Problems in Engineering
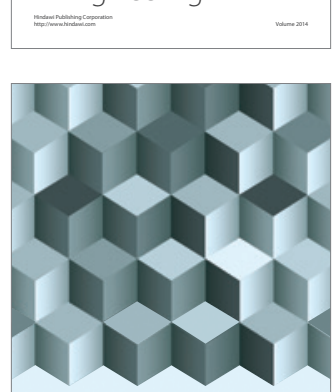

Journal of

Function Spaces
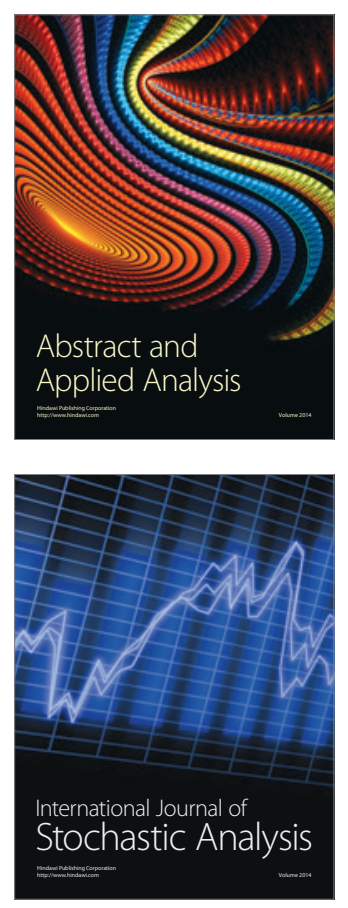

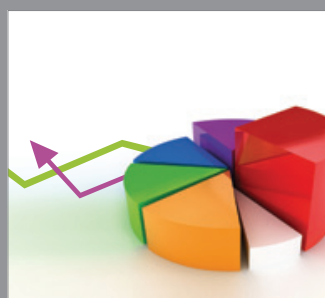

ournal of

Probability and Statistics

Promensencen
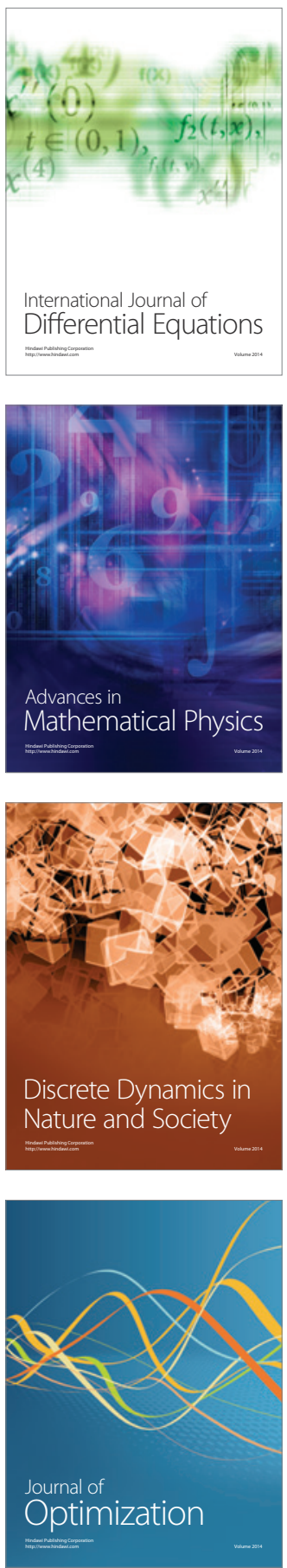